\title{
Tunable apodizers and tunable focalizers using helical pairs
}

\author{
Jorge Ojeda-Castaneda, ${ }^{1}$ Sergio Ledesma, ${ }^{1}$ and Cristina M. Gómez-Sarabia ${ }^{2}$ \\ ${ }^{I}$ Electronics Department, University of Guanajuato, Salamanca 36885, México, \\ ${ }^{2}$ Digital Arts, Department, University of Guanajuato, Salamanca 36885, México
}

Received March 9, 2013; accepted March 27, 2013, published March 31, 2013

\begin{abstract}
We unveil the use of two helically distributed amplitude masks for controlling the damping coefficient of Gaussian-like windows. We show that by introducing an in-plane rotation between the members of the proposed pair, one eliminates the polar dependence of the masks. Then, one can tune the half-width of radial Gaussian-like apodizers. In a similar manner, we show that if one uses two helically distributed refractive elements, as a pair, then one can control the maximum value of the optical path difference; which is useful for tuning several radial focalizers.
\end{abstract}

Certain amplitude masks are useful for expanding the axial irradiance distribution [1-3]. Other phase masks are able to reduce the influence of focus errors on the Modulation Transfer Function (MTF) [4-9]. However, these phase masks generate unwanted oscillation around a tendency curve. A tunable Gaussian apodizer can reduce these undesirable oscillations [10].

According to Plummer, Baker and van Tassel [11], variable optical power lenses were first suggested by Kitajima [12] and later on by Birchall [13]. Lohmann [1417] and Alvarez [18, 19] re-discovered independently Kitajima's technique for implementing varifocal lenses. By employing helical wavefronts, one can generate variable Fresnel patterns [20, 21], as well as vortex lenses [22].

Here, our aim is twofold. First, we present the use of a pair of helical distributed amplitude masks for tuning the damping coefficient of Gaussian-like windows. Second, we show that by using two suitable helical refractive elements, one can control the optical path difference of radial focalizers.

In Fig. 1 we depict schematically the optical system under discussion. In what follows we describe two amplitude masks that form a pair, which acts at the pupil aperture. By employing polar coordinates, the amplitude transmittance of the first amplitude mask is

$$
\mathrm{R}_{1}(\rho, \varphi)=\mathrm{e}^{-2 \pi \mathrm{c}\left(1+\frac{\varphi}{2 \pi}\right) \mathrm{F}(\rho)} \operatorname{circ}\left(\frac{\rho}{\Omega}\right)
$$

*E-mail: jorge_ojedacastaneda@yahoo.com
In Eq. (1) the Greek letter $\rho$ represents the radial spatial frequency variable, whose maximum value is the cut-off spatial frequency $\Omega$. Hence, the transparent circular aperture is denoted as $\operatorname{circ}(\rho / \Omega)$. The Greek letter $\varphi$ is the polar angle. The lower case letter " $\mathrm{c}$ " is a dimensionless damping factor. The radial variation of the mask is represented by the real function $F(\rho)$; such that $0 \leq F(\rho) \leq 1$.

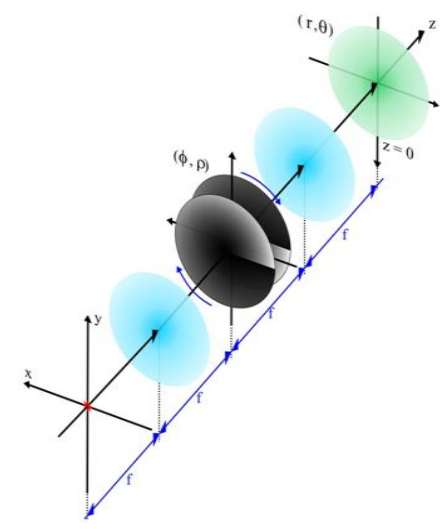

Fig. 1. Schematic diagram of the optical setup.

In a similar fashion, the amplitude transmittance of the second mask is

$$
\mathrm{R}_{2}(\rho, \varphi)=\mathrm{e}^{-2 \pi \mathrm{c}\left(1-\frac{\varphi}{2 \pi}\right) \mathrm{F}(\rho)} \operatorname{circ}\left(\frac{\rho}{\Omega}\right)
$$

It is worth noting that

$$
\lim _{\varphi \rightarrow 0} R_{1}(\rho, \varphi)=\lim _{\varphi \rightarrow 0} R_{2}(\rho, \varphi) \leq 1 .
$$

And that

$$
\mathrm{R}_{2}(\rho, 2 \pi)=1
$$

Now, we form a pair by placing the two previously described masks in contact. If we introduce an in-plane rotation between the elements of the pair, say by an angle $\beta$, then the amplitude transmittance is 


$$
\mathrm{T}(\rho ; \varphi)=\mathrm{R}_{1}\left(\rho, \varphi+\frac{\beta}{2}\right) \mathrm{R}_{2}\left(\rho, \varphi-\frac{\beta}{2}\right) .
$$

By substituting Eqs. (1) and (2) in Eq. (4) we obtain

$$
\mathrm{T}(\rho ; \beta)=\mathrm{e}^{-2 \pi \mathrm{c}\left(2+\frac{\beta}{2 \pi}\right) \mathrm{F}(\rho)} \operatorname{circ}\left(\frac{\rho}{\Omega}\right)
$$

From Eq. (5) we have that the overall amplitude transmittance is independent of the polar angle $\varphi$.

a)

b)

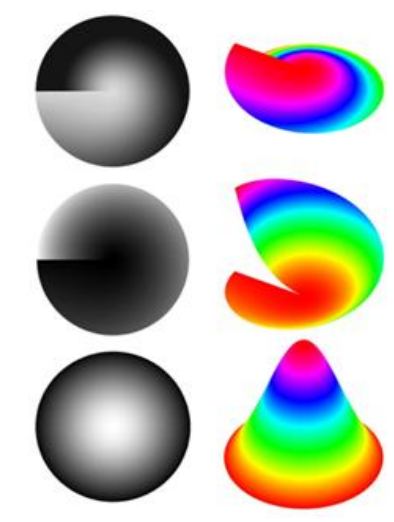

Fig. 2. Gray level pictures and amplitude transmittances: a) first element, b) second element, c) the pair.

Furthermore, one can control the damping factor of the pair, simply by changing the value of $\beta$. In Fig. 2 we display the gray level pictures, as well as the plots of Eqs. (1), (2) and (5). For the pictures at the bottom of Fig. 2, we consider that $\beta=\pi / 10$; and we illustrate the control of a Gaussian profile, $F(\rho)=(\rho / \Omega)^{2}$. We note here that our proposed pair is equally useful for controlling radially distributed hyper Gaussian masks,

$$
\mathrm{T}(\rho ; \beta)=\mathrm{e}^{-2 \pi \mathrm{c}\left(2+\frac{\beta}{2 \pi}\right)\left(\frac{\rho}{\Omega}\right)^{\mathrm{s}}} \operatorname{circ}\left(\frac{\rho}{\Omega}\right),
$$

as well as for annularly distributed masks. For this latter case, Eq. (5) becomes

$$
P(\xi ; \beta)=e^{-2 \pi c\left(2+\frac{\beta}{2 \pi}\right) \xi^{s}},
$$

where $\zeta=(\rho / \Omega)^{2}-0.5$. In Eqs. (6) and (7) the Latin letter $" s "$ is any real number. Hence, the following clarification is in order. If we set $s=2$ in Eq. (6) we have a Gaussian attenuating mask. For $0<\mathrm{s}<2$, we have a subgaussian mask. And if $2<\mathrm{s}$, then we have a supergaussian mask.
The same notation applies for the annularly distributed masks in Eq. (7).

Next, we present the complex amplitude transmittances of two refractive elements, whose phase delays are helically distributed. We show that by rotating the proposed elements, one can implement tunable versions of axicons, lenses and axilenses. Again, by using polar coordinates the complex amplitude transmittance of the first refractive element is

$$
\mathrm{R}_{1}(\rho, \varphi)=\mathrm{e}^{\mathrm{ia} \varphi \mathrm{G}(\rho)} \operatorname{circ}\left(\frac{\rho}{\Omega}\right) .
$$

In Eq. (8) the lower case letter "a" is a maximum value of the optical path difference. As before, the Greek letter $\varphi$ is the polar angle. The radial phase variation is described by the real function $G(\rho)$; such that $0 \leq \mathrm{G}(\rho) \leq 1$. The complex amplitude transmittance of the second refractive element is

$$
\mathrm{R}_{2}(\rho, \varphi)=\mathrm{e}^{-\mathrm{ia} \varphi \mathrm{G}(\rho)} \operatorname{circ}\left(\frac{\rho}{\Omega}\right)
$$

We form a refractive pair; by placing in contact the two previously described elements. If we introduce an in-plane rotation; say by an angle $\beta$ between the elements, the complex amplitude transmittance of the proposed pair is

$$
\mathrm{T}(\rho ; \beta)=\mathrm{R}_{1}\left(\rho, \varphi+\frac{\beta}{2}\right) \mathrm{R}_{2}\left(\rho, \varphi-\frac{\beta}{2}\right) .
$$

From Eqs. (8), (9) and (10), we obtain that

$$
\mathrm{T}(\rho ; \beta)=\mathrm{e}^{\mathrm{ia} \beta \mathrm{G}(\rho)} \operatorname{circ}\left(\frac{\rho}{\Omega}\right) .
$$

From Eq. (11) we recognize that the pair is able to generate a complex amplitude transmittance that is independent of the polar angle $\varphi$. It is also apparent from Eq. (11) that one can control the maximum value of the optical path difference by changing the value of $\beta$.

For illustrating the latter result we consider the following examples. For an axicon [23] the function $\mathrm{G}(\rho)$ is

$$
G(\rho)=(\rho / \Omega) .
$$

Of course, for a lens we have that

$$
G(\rho)=(\rho / \Omega)^{2} .
$$

And finally, for an axilens [24] we have that 


$$
\mathrm{G}(\rho)=\frac{(\rho / \Omega)^{2}}{1+(\rho / \Omega)^{2}}
$$

In Fig. 3, along the first column, we plot the phase profiles in Eq. (12), (13) and (14). We note that since the second phase mask is the complex conjugate of the first mask, it is sufficient to visualize only one element of the pair. In Fig. 3, along the second column, we display the interferograms associated with one element of the pair.

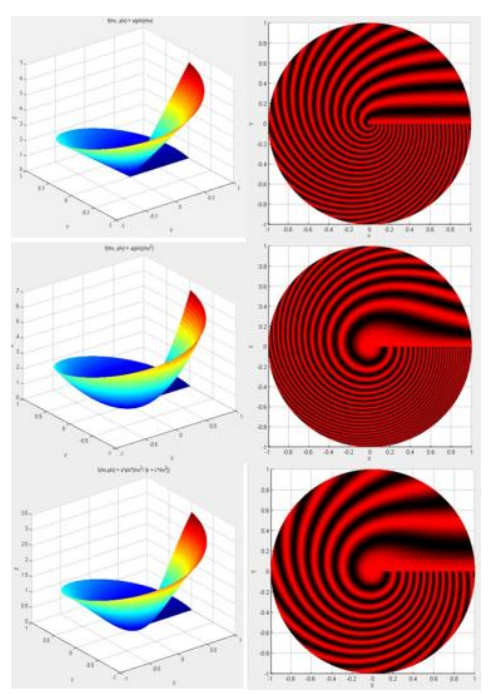

Fig. 3. Helical versions, and interferograms of: a) an axicon, b) a lens, and c) an axilens.

For the plots along column 1, in Eq. (11), we set $\mathrm{a}=1$. However, for the interferograms we employ the value $\mathrm{a}=$ 25.

Summarizing, first, we have presented the use of two attenuating masks, which have helically distributed amplitude variations. We have proposed to use two masks for forming a pair. We have shown that by introducing an in-plane rotation between the elements of the pair, one can control the damping coefficient of almost any radially varying profile. By tuning the damping coefficient, one controls the half-width of the Gaussian apodizers. We have noted that the proposed procedure is useful for tuning subgaussian windows or supergaussian windows. We have also noted that the proposed device is useful for tuning apodizers that have annularly varying profiles.

Second, we have discussed the use as a pair two refractive elements which have helically distributed phase variations. We have noted that the complex amplitude transmittance of the first element is the complex conjugate of the transmittance associated with the second element. We have shown that by introducing an in-plane rotation between the elements of the pair, one can control the maximum value of the optical path difference, of almost any radial phase variation. Hence, we have proposed the use of the refractive pair (whose elements have helically distributed phase variations) for implementing tunable axicons, lenses, and axilenses.

We gratefully acknowledge the financial support of CONACYT (157276) and PROMEP (103.5/10/46.12, and PTC-197 D).

\section{References}

[1] W.T. Welford, J. Opt. Soc. Am. 50, 749 (1960).

[2] J. Ojeda-Castañeda, L.R. Berriel Valdos, E.L. Montes, Opt. Lett. 8(8), 458 (1983).

[3] J. Ojeda-Castañeda, L.R. Berriel-Valdos, E. Montes, Appl. Opt. 26, 2770 (1987).

[4] E. R. Dowski, T. W. Cathey, Appl. Opt. 34, 1859 (1995).

[5] A. Sauceda, J. Ojeda-Castañeda, Opt. Lett. 29, 560 (2004).

[6] A. Castro, J. Ojeda-Castañeda, Appl. Opt. 43, 3474-3479 (2004).

[7] S. Mezouari, G. Muyo, A.R. Harvey, J. Opt. Soc. Am. A 23, 1058 (2006).

[8] G. Mikuła, Z. Jaroszewicz, A. Kolodziejczyk, K. Petelczyc, M. Sypek Opt. Exp. 15, 9184 (2007).

[9] J. Ojeda-Castañeda, J.E.A. Landgrave, C. M. Gómez-Sarabia, Appl. Opt. 47, E99 (2008).

[10] J. Ojeda-Castaneda, E. Yépez-Vidal, E. García-Almanza, C.M. GómezSarabia, Phot. Lett. Poland 4 (3), 115 (2012).

[11] W.T. Plummer, J.G. Baker, J. van Tassell, Appl. Opt. 38, 3572 (1999).

[12] I. Kitajima, British Patent 250, 268 (July 29, 1926).

[13] H.J. Birchall, U. S. Patent 2,001, 952 (May 21, 1935).

[14] A.W. Lohmann, British Patent 998, 191 (May 29, 1964).

[15] A.W. Lohmann, Republic Française 1, 398, 351 (June 10, 1964).

[16] A.W. Lohmann, Italy 727, 848 (June 19, 1964).

[17] A.W. Lohmann, Appl. Opt. 9, 1669 (1970).

[18] L.W. Alvarez, U. S. Patent 3, 305, 294 (February 21, 1967).

[19] L.W. Alvarez, W.E. Humphrey, U. S. Patent 3, 507, 565 (April 21, 1970).

[20] A.W. Lohmann, IBM Technical Disclosure Bulletin 10, 407 (1967).

[21] A.W. Lohmann, Appl. Opt. 6, 1567 (1967).

[22] K. Crabtree, J.A. Davies, I. Moreno, Appl. Opt. 43, 1360 (2004).

[23] J.H. McCleod, J. Opt. Soc. Am. 44, 592 (1954).

[24] N. Davidson, A.A. Friesem, E. Hasman, Opt. Lett. 16, 523 (1991). 